\title{
Human upper limb manipulator mass center motion and mass moments of inertia variation
}

\author{
Gergana Nikolova, ${ }^{*}$, Daniel Dantchev, and Alexander Kazakoff \\ Institute of Mechanics, Bulgarian Academy of Sciences, Acad. G. Bonchev Str., Building 4, \\ Sofia 1113, Bulgaria
}

\begin{abstract}
Motion control is complicated for people having traumas or neurological diseases. An underlying assumption in our work is that the motion of healthy people is optimal with respect to positioning accuracy, movement response, and energy expenditure. In this paper, a new approach for determination of the human upper limb mass-inertial characteristics is presented by using the 3D geometrical mathematical modeling analysis approach. Two examples will be given to illustrate the main features and advantages of the proposed design concepts. The objective of the work presented in this paper is a determination of the mass properties of a two joints human upper limb manipulator. Results are aimed to have application in an exoskeleton design, the design of manipulation system and external manipulation system, serving people with some motion difficulties, as well as in sport and rehabilitation.
\end{abstract}

\section{Introduction}

A new modified biomechanical geometrical model of the human upper limb is presented, with the help of three-dimensional geometrical mathematical modeling 3D. This model represents a modification of the ones suggested in [1,2] and is based on our own anthropometric measurements of 50 men aged between 30-40 years. Basic anthropometric dimensions were reported before [3, 4]. The investigation on the human body geometric and mass-inertial characteristics is of key importance in human motion analysis. This investigation is a natural continuation of the work, presented in [5]. The objective of the work presented in this paper is a definition of the mass properties of a two joints human upper limb manipulator. Two examples of two different positions of the upper limb are presented in order to illustrate the main features and advantages of the proposed design concept. The two positions are

\footnotetext{
*Corresponding author: gergana1973@gmail.com
} 
depicted in Figs 2 and 3. We define a coordinate frame $\boldsymbol{F}$ with its origin at the midpoint of the shoulder joint with the z-axis along the body vertically downwards, the $\mathrm{x}$-axis in the sagittal plane, and the y-axis is in the transversal plane. For any of the two positions we present the coordinates of the center of mass, the principal moments of inertia, the components of the unit vectors that define the coordinate frame in which the inertial tensor is diagonal, and the moments of inertia with respect to the $\boldsymbol{F}$, as well as its components with respect to a system that has its center at the center of mass but with axes aligned with $\boldsymbol{F}$. Finally, we present data how the center of mass of the upper limb changes when it moves so that its end reaches the area of the mouth of the human - one imagines a movement of a disabled person who shall be helped in order to drink a cap of water, eat a meal, etc.

The objectives of the work presented in this paper have been achieved by fulfilling the following main tasks:

1) building up a $3 \mathrm{D}$ geometrical mathematical model of the upper human limb manipulator, using the statistical data presented in [3, 4];

2) Secondly, verification of the proposed model via comparison of the data obtained from the determination of the human upper limb manipulator mass properties by using the computer code and those derived with the analytical means from the previous studies. The comparison demonstrated a nice agreement between these data.

3) Investigation on how the center of mass of the proposed manipulator changes with respect to angle variations in two planes: $i$ ) one defined by the plane of the manipulation system and ii) the second one, motion of this particular plane with respect to the origin of the coordinate system, situated in the human shoulder, i.e., with respect to the laboratory coordinate frame.

4) Visualization of the mass center motion in 3D space, which is expected to be a partial saddle like surface;

5) Derivation of data that can be used in the design of devices aimed to help disabled people, having problems with the movement of their upper limbs.

We consider two joints model of the upper limb. We take that the upper arm can move only in the $(\mathrm{z}, \mathrm{x})$ plane with an angle $\alpha$ that characterizes its position. The lower arm is taken to be able to move in the plane formed by the upper and the lower arm with angle $\beta$ determining the position of one with respect to the other one. The proposed topic seems to be very interesting and attracting the attention of many scientists worldwide. For example, investigations on a human-arm-like mechanical manipulator are reported before $[5,6,8,9,10]$. Reference [7] can be used as a general source of knowledge on the subject. In addition, upper limb portable motion analysis system, based on inertial technology for neuro-rehabilitation purposes is revealed in [11]. Reference [12] deals with control of hand equilibrium trajectories in multi-joint arm movements. In [13] one investigates the coordination of arm movements and present there an experimentally confirmed mathematical model. The effect of visuomotor displacements on arm movement paths is presented in [14]. Finally, the mechanics of multi-joint posture and movement control are revealed in Ref. [15]. 


\section{Model building up}

The ideology of the 3D geometrical mathematical modelling - 3D (GMM) analysis is a creation of the geometrical model of the investigated body and by applying on the geometry, the material density and the analytical properties of the $3 \mathrm{D}$ bodies used in the approximation to determine all properties of interest including the body mass properties and the mass moments of inertia, respectively. The model geometry of human upper limb presented in Fig. 1. Fig 1a) represents the cross-section of the upper part of the arm at its upper anthropometric point, Fig. 1b) - the corresponding cross-section where the upper arm meets the lower arm, and Fig. 1c) - the crosssection of the lower end of the lower arm. We recall that the upper arm, that extends between acromion-radiale anthropometric points, while the lower arm is taken to be, as usual, between the points radiale-stylion. The cross sections shown in Fig. 1 are known under the name stadium solids. Its cross-section consists of a rectangular + two parts of ellipsis on the left and right-hand sides. The radii of the ellipsis are such that one has the width and the proper circumference of the crosses section, which are measured for the set of individuals considered.

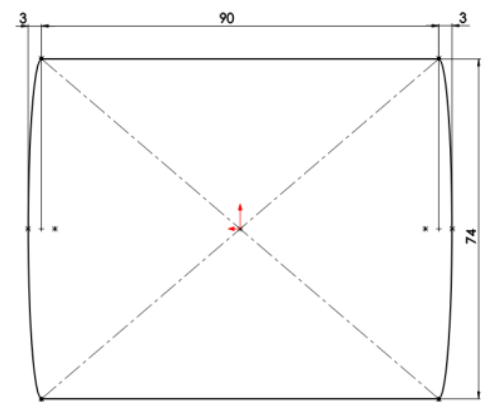

a) Upper Arm upper surface;

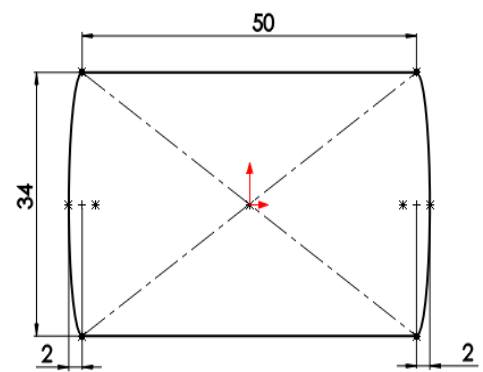

c) Lower Arm lower surface;

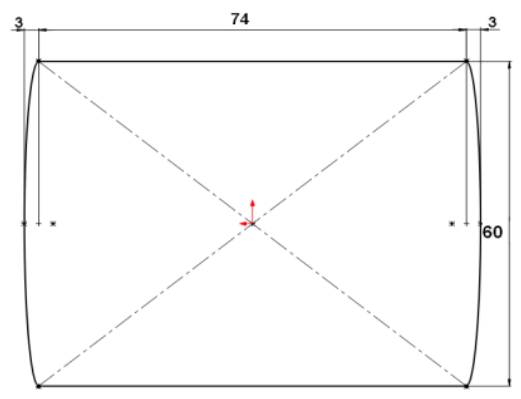

b) Upper Arm lower surface;

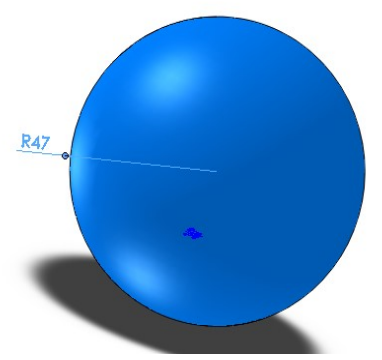

d) Human hand modeled like sphere;

Fig. 1. Sketches for human upper limb and hand, modeled as a sphere - basic dimensions in $\mathrm{mm}$. 
The full model geometry, presented in Figs 2 and 3, can be achieved by extruding the plane figures 1a) and $1 \mathrm{~b}$ ) to obtain the upper arm and figures $1 \mathrm{~b}$ ) and 1c) to form the lower arm of the upper limb with lengths $309 \mathrm{~mm}$ and $247 \mathrm{~mm}$, respectively [16]. The model mass properties and the corresponding mass moments of inertia can be computed applying the corresponding material density on the model geometry. The calculated results for the three human upper limb parts are compared with the analytically already calculated and reported in [5] mass moments of inertia. The agreement between the computer model and the analytical results for any of the segments separately gives us confidence that the model can be used for determining the mass inertial characteristic of the whole upper limb for any its special position.

\section{Results from the model}

The role of the angles $\alpha$ and $\beta$ and the principal positions of the upper limb in our model are depicted in Figs. 2 and 3. The Fig. 3 demonstrates the role of the angle $\beta$ for the movement of the lower arm when the upper arm is simply kept fixed aligned with the body along the z-axis. Axes 1-5 in Figs. 2 and 3 are automatically created by the computer system and represent three axes in the basic co-ordinate system $X$, $\mathrm{Y}, \mathrm{Z}$ with origin in the shoulder and the other two are oriented longitudinally along the upper and the lower arm, respectively.

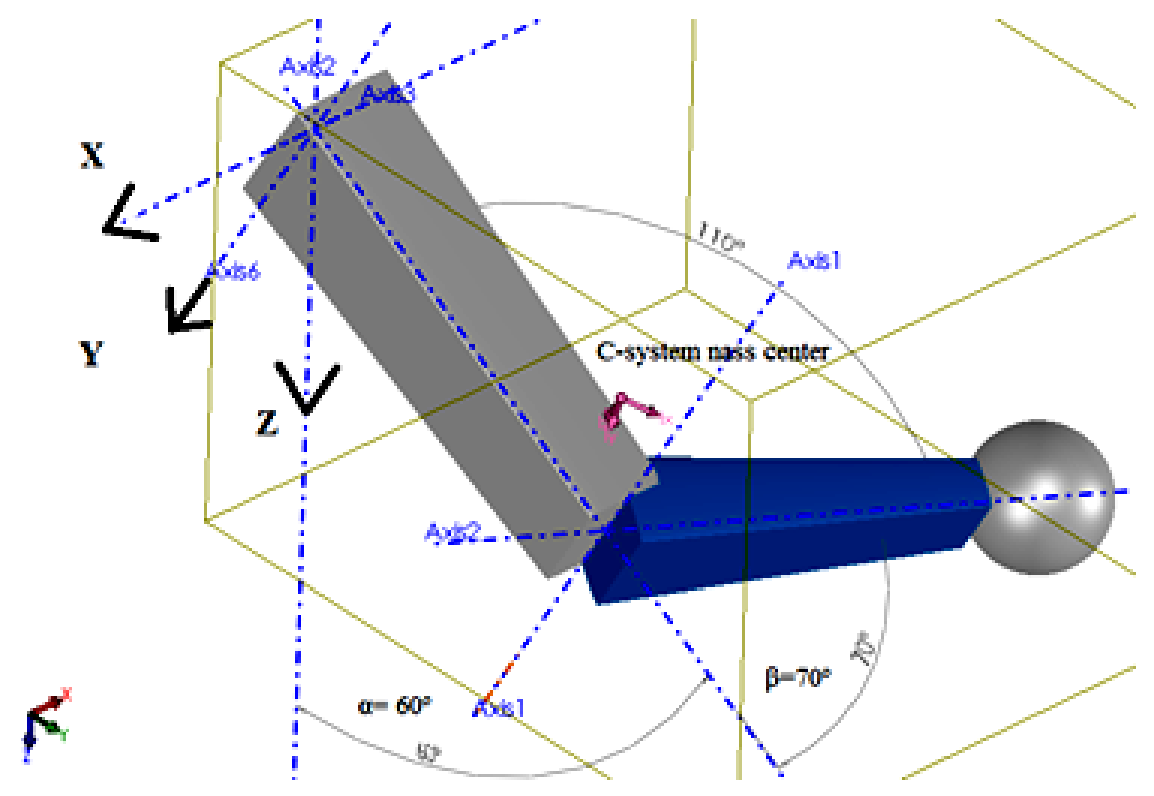

Fig. 2. Manipulator with two joints, position $\alpha=60^{\circ}, \beta=70^{\circ}$.

Before reporting the mass inertial parameters for the upper limb in the above positions let us state that our computer model generated in CAD media SolidWorks, has been verified against analytical calculation of the mass inertial parameters for 
any of the components of the upper limb which includes the volume, mass, position of center of mass and the inertial moments of the components [5]. That is why we have a confidence that it reliably works for a general position of the upper limb. In principle, our computer model can provide data for mass, volume, surface area, center of mass-coordinates, principal axes of inertia and principal moments of inertia, moments of inertia taken in a coordinate frame at the center of mass and axes aligned with the output coordinate system and moments of inertia, taken at the output coordinate system. For example, this data for the upper limb position shown in Fig. 2 are:

Mass $=3248.13 \mathrm{~g}$, Volume $=3015170.96 \mathrm{~mm}^{3}$, Surface area $=193039.49 \mathrm{~mm}^{2}$, Center of mass coordinates (in mm): $C_{x}=-67.97, C_{y}=-205.80, C_{z}=-205.80$. The unit vectors defining the principal axes of inertia are with components $\vec{e}_{1}=(0.59,0.70,-0.40), \vec{e}_{2}=(-0.81,0.51,-0.30)$, and $\vec{e}_{3}=(0.00,0.50,0.87)$, and the principal moments of inertia: $\left(\mathrm{kg} \times \mathrm{dm}^{2}\right)$ are

$$
P_{x}=1.25, P_{y}=8.3, P_{z}=9.2
$$

Moments of inertia taken at the center of mass and aligned with the output coordinate system are:

$$
\begin{array}{ccc}
L x x=5.8 & L x y=2.9 & L x z=-1.7 \\
L y x=2.9 & L y y=5.1 & L y z=-2.4 \\
L z x=-1.7 & L z y=-2.4 & L z z=7.9
\end{array}
$$

while moments of inertia taken at the output coordinate system are:

$$
\begin{array}{ccc}
I x x=24.2 & I x y=7.5 & I x z=-4.3 \\
I y x=7.5 & \text { Iyy }=11.2 & \text { Iyz }=-10.3 \\
I z x=-4.3 & \text { Izy }=-10.3 & \text { Izz }=23.1
\end{array}
$$

The point $\mathrm{C}$ represents the center of mass of the manipulator in the configuration given - see Figs 2 and 3. It also is the origin of the coordinate system shown in Figs 2 and 3, in which the inertial moments tensor is in its diagonal form. Similar detailed data can be reported for any position of the upper limb. For example, for the configuration shown in Fig. 3 one obtains:

$$
C_{x}=-36.16, C_{y}=0, C_{z}=150.26
$$

Using the possibilities offered by the model we have determined also how the center of mass of the upper limb changes when it moves in a way that the hand reaches near the mouth of the human. Fig. 4 shows the C-mass center-coordinates in $[\mathrm{mm}]: \alpha$ varies with seven values from zero to $60^{\circ}$, while $\beta$ varies with eleven values from $70^{\circ}$ to $170^{\circ}$. A saddle like surface of the positions of the center of mass is defined in this way. 


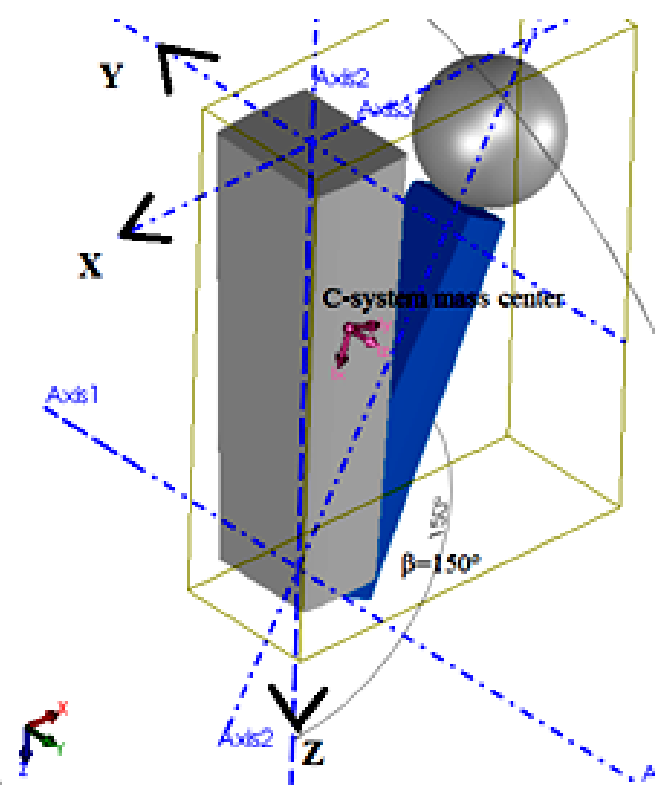

Fig. 3. Manipulator with two joints- $\alpha=0^{\circ}$ and $\beta=150^{\circ}$.

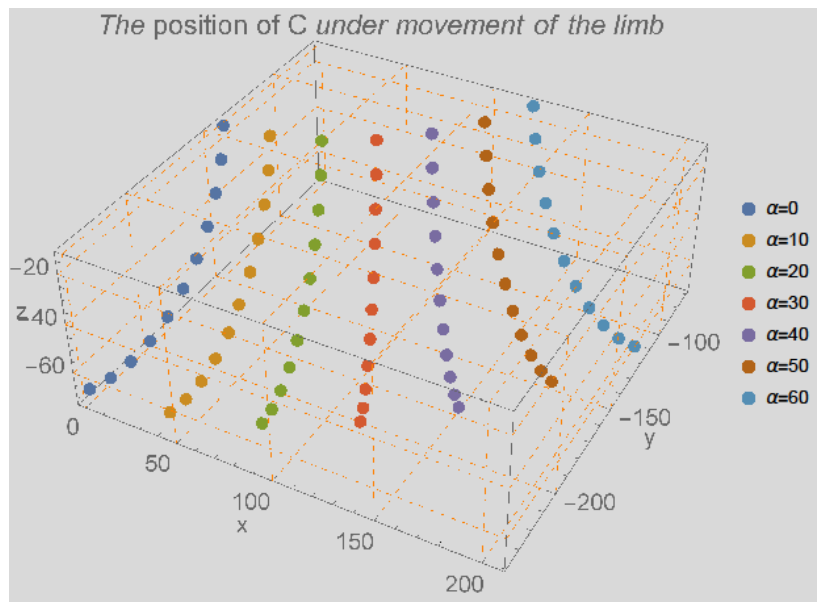

Fig. 4. Graphics of mass center of motion-coordinates in [mm].

\section{Conclusion}

In this paper, a new approach for determination of the human upper limb massinertial characteristics is presented by using the $3 \mathrm{D}$ geometrical mathematical modeling analysis approach. Results are presented, derived from the built-up 3D geometrical mathematical analysis, demonstrating few of the options offered by our approach. Analysing the results, defined by the new approach, the following conclusions can be stated along the z-axis. 
1. A new 3D geometrical mathematical model of the human upper limb is build up, utilizing a computer code.

2. Mass properties of the human upper limb manipulator are determined and partially illustrated in the current paper.

3. A comparison is performed with the mass properties, defined by the analytical analysis and reported before in previous studies. The achieved precision is perfect and this fact allows us future calculations, using this $3 \mathrm{D}$ model to be performed with confidence.

4. Visualisation of the manipulation mass center motion is performed under the variation of both investigated angles. A partial saddle like surface is generated, defining the motion in the original laboratory coordinate system.

5. Data are generated, that can be used in design of devices aimed to help disabled people having problems with the movement of their upper limbs.

\section{Future work}

An obvious direction of future activities is the improvement of the proposed model so, that the geometrical modelling becomes as close as possible to the real shape of the human upper limb. In the current study we used the geometrical parameters directly as measured from a set of individuals. The approximation with specific 3D bodies of the segments of the limb leads to a given mistake in reproducing its mass. If one insists of using the physically measured density one way around that problem is to modify with, say, $5 \%$ precision the actual measure data in such a way that the obtained mathematical model reproduces exactly the mass of segments of the limb as follows from the corresponding regression equations. Since they do depend on the gender of the corresponding person in such a way our model will also become, to a given extend, gender specific.

Authors believe that the presented study can be really helpful in the design of manipulation systems with application in sport and rehabilitation providing the handy opportunity to calculate and determine the mass-inertial parameters of the upper limb of a given individual provided the anthropometric easily measurable data for that individual are known.

The financial support by the Bulgarian National Science Fund: Contract DN-07/5 "Study of anthropometric and mass-inertial characteristics of the Bulgarian men and women via mathematical models of the human body" is gratefully acknowledged.

\section{References}

1. G. Nikolova, Y. Toshev, J. Biomech., 40, 3700-3707 (2007).

2. G. Nikolova, J. Vibroeng., 11, 2, 274-278 (2009).

3. G. Nikolova, J. Theoretical Appl. Mech., 40, 3, 101-116 (2010).

4. G. Nikolova, J. Theoretical Appl. Mech., 40, 4, 73-80 (2010).

5. G. Nikolova, D. Dantchev, Al. Kazakoff, Proc. of the 3-rd World Congress on New Technologies (NewTech'17) Rome, Italy, 2017, (to be published).

6. V. Grecu, N. Dumitru, L. Grecu, Proc. of the International Multi Conference of Engineers and Computer Scientists, Vol II, IMECS, Hong Kong (2009). 
7. Ph. Ringrose, M. Bemtley, Springer Dordrecht Heidelberg New York London, ISBN 978-94-007-5496-6, DOI 10.1007/978-94-007-54973(eBOOK).

8. F. J. Valero-Cuevas, DOI 10.1007/978-1-4471-6747-1_2, Springer-Verlag London, 2016.

9. M. Z. Al-Faiz, A. F. Shanta, Intelligent Control and Automation, 6, 29-37 (2015).

10. A. Hemami, Robotica, 5, 23-28 (1987).

11. R. Perez et all, Sensors, 10, 1, 0733-10751 (2010).

12. T. Flash, Biol. Cybern, 57, 257-274 (1987).

13. T. Flash, N. Hogan, The Journal of Neuroscience, 5, 7, 1688-1703 (1985).

14. S. J. Goodbody, D. M. Wolpert, Exp Brain Res, 127, 213-223, (1999).

15. N. Hogan, Biol. Cybern, 52, 315-331 (1985).

16. Y. Yordanov et al. Anthropology of the Bulgarian population at the end of the 20th century (30-40 years old persons), Prof. M. Drinov Publishing House, Sofia, (2006). 